\title{
The Cursor as an Artistic Expression in Jeddah
}

\author{
Maha Zeini Al-Saati, Jim Bizzocchi, and David Botta \\ Simon Fraser University, School of Interactive Arts and Technology, \\ 250 - 13450 - 102 Ave, V3T 0A3, Surrey, BC, Canada \\ \{malsaati, jimbiz, botta\} asfu.ca
}

\begin{abstract}
The paper discusses Jeddah, an interactive artwork that experiments with the relation between cursor and background art. In addition to being functional, the cursor is also cast as an element of artistic expression. It is exaggerated in shape, scale, color and motion to blend in with the background art, and is constantly transforming in response to the art world. This is done to enhance the mood and experience of spatial exploration of Jeddah's world.
\end{abstract}

Keywords: Cursor transformation, cursor aesthetics, spatial exploration, experimental artwork.

\section{Introduction}

Jeddah portrays an historic neighborhood in Saudi Arabia, with buildings that were once beautiful, now decaying or demolished. It places the user in the first person perspective, to unfold the narrative through spatial exploration. To complement the artwork, an expressive cursor blends and interacts with the background's artwork, exaggerated in shape, scale, color and motion, transforming with its changing position [1]. The background art consists of stylized real photographs that mimic media forms such as collage and water-colored paintings. This is done to establish a dream-like mood, metaphor and character [2], [3].

\section{Jeddah's Expressive Cursor}

Jeddah's aesthetic approach to interface design adopts Shamonsky's [4] position that the ease-of-use in today's functionally designed interfaces takes away the excitement of interaction with an artifact. The aesthetic approach encourages the user to sensually engage with the interface itself, as opposed to the user purely focusing on the task at hand. Interfaces can also become actors in the narrative, acting as extensions of the user, like a gun becoming an extension of the user's hand in first-person shooter games [5], [6], [7], [8], [9]. In Jeddah, the cursor serves as a functional and guidance tool, as well as one of artistic expression. The expressive cursor melts into the textures and attitudes of the story world, while also guiding the user by hinting the consequences of a click, thereby breaking the norm of point-and-click [1]. 


\subsection{Cursor as Functional Tool}

The cursor indicates possible actions when rolling over hotspots, while the nature of the highlight indicates how the action fits with space. For example, upon rolling over electrical wires in the scene, the cursor changes to a hand to grab the wires and transport to another location. When flight is possible, it gracefully transforms from the initial breathing spiral to a winged figure with its own sense of function and narrative impact.

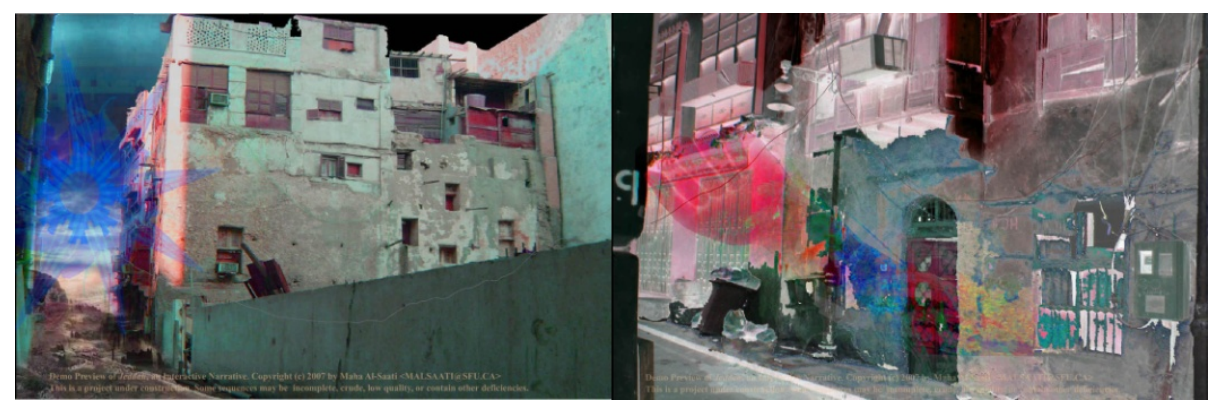

Fig. 1. The cursor transforms into a rotating star (left) or a hand (right) to indicate action

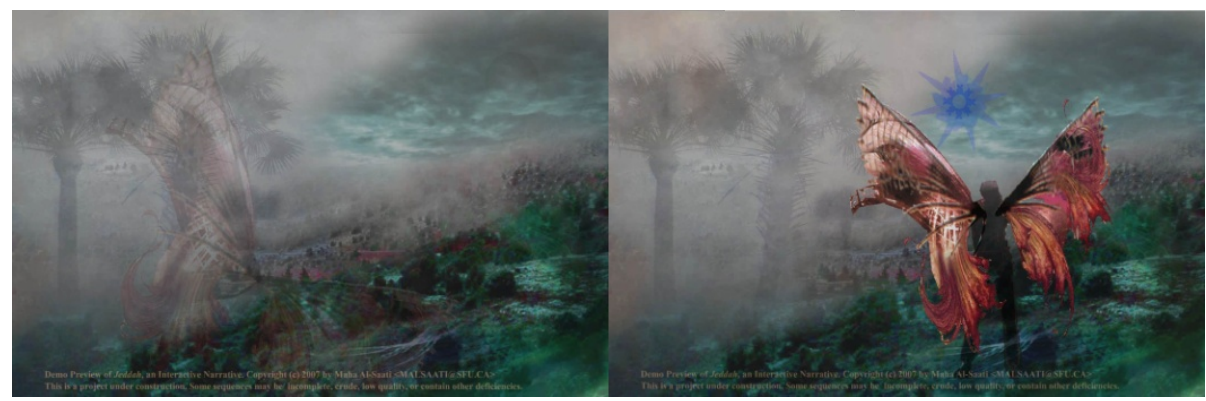

Fig. 2. The cursor gracefully transforms from a butterfly (left) to a winged figure and finally a rotating star (right)

\subsection{Cursor as Guidance Tool}

The cursor acts as a guide throughout the work by signaling possible actions. It transforms in shape, scale, color and motion to foreshadow the consequence of the click. When arriving at a forking path, hovering the cursor on different areas gives an impression of the destination ahead. This effect was achieved by layering several backgrounds, and changing their transparency values in response to the cursor's position. Pointing the cursor towards the sky transforms it into a butterfly to indicate flight, while pointing it towards the land transforms the landscape into a mirage or desert. 


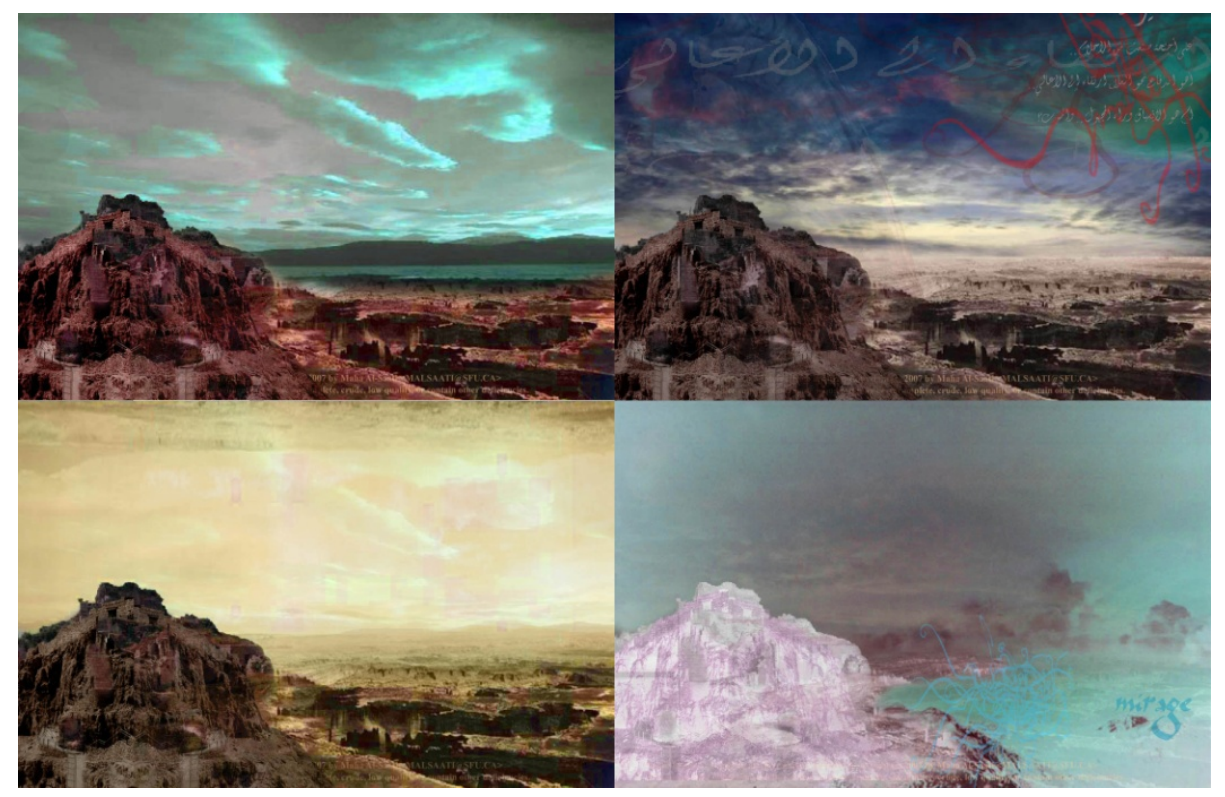

Fig. 3. The position of the cursor on the screen foreshadows the eventful consequence of clicking in that direction by changing the entire background to emphasize the sky, the desert land, or a mirage

\subsection{Cursor as Artistic Expression}

The cursor's design complements the artwork and narrative of Jeddah. Its scale is initially exaggerated so that it covers the whole screen at times; yet, it is also translucent, blending within the story world. It expands and rotates to imitate a breathing motion, changing shape to mimic the actions which it represents, acting as an extension of the user. Other moving objects in the scenes serve to complement the artistic composition and advance an ongoing narrative. The cursor is transformed by the actions of these objects that include: mist, wires, nightmarish figures, and calligraphic poetry floating in the air.

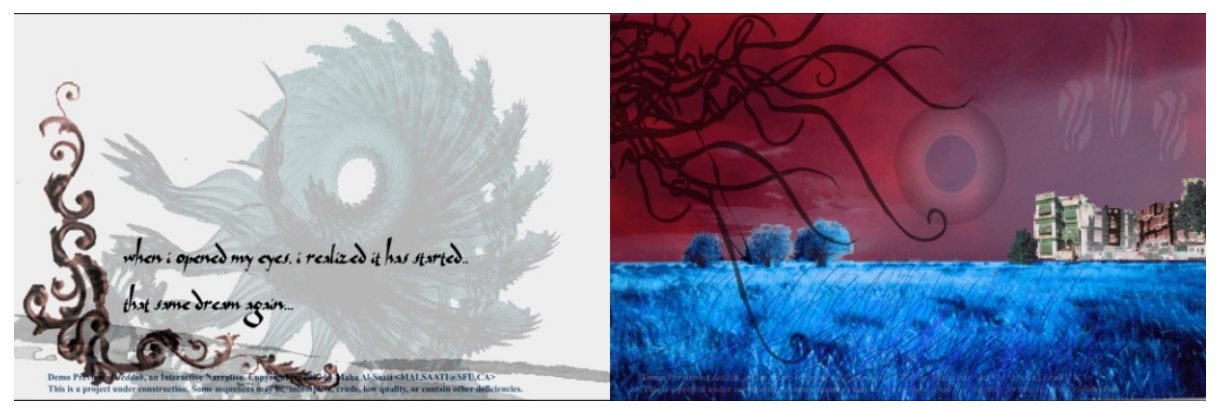

Fig. 4. The cursor is an artistic element that complements the background. It is seen as a rotating spiral (left), or a breathing spirit attacked by a nightmarish figure (right). 


\section{Discussion}

This paper suggests the use of cursor as an aesthetic as well as functional device in interactive artworks. It outlines the concepts behind the creation of Jeddah's cursor, treated as both a functional guide and an expressive actor. Through the use of expressive treatments, the cursor enhances the experience of narrative, mood and spatial exploration in Jeddah's interactive artwork.

\section{References}

1. Bizzocchi, J.: Ceremony Of Innocence and the Subversion of Interface: Cursor Transformation as a Narrative Device. In: 5th International Digital Arts and Culture Conference, MelbourneDAC 2003, Melbourne (2003)

2. Fischer, J., Haller, M., Thomas, B.: Stylized Depiction in Mixed Reality, Stylized Depiction. The International Journal of Virtual Reality 7(4), 71-79 (2008)

3. McGandy, M.J., Seligmann, D.D.: Multimedia Enlightenment: Transcendent Experiences with the Help of Technology. In: IEEE Multimedia, vol. 13(1), pp. 4-8. Norton Professional Books (2006)

4. Shamonsky, D.J.: Poetic Interfaces (2007), http://architecture.mit.edu/ dorothy/papers/poetic.html

5. Fishwick, P.: Aesthetic Computing. MIT Press, Cambridge (2006)

6. Bertelsen, O., Pold, S.: Towards the Aesthetics of Human-Computer Interaction. In: Proceedings of the Second Danish Human-Computer Interaction Research Symposium, pp. 11-12. University of Copenhagen, Copenhagen (2002)

7. Bertelsen, O., Pold, S.: Criticism as an Approach to Interface Aesthetics. In: Proceedings of the Third Nordic Conference on Human-Computer Interaction, pp. 23-32. ACM, New York (2006)

8. Pold, S.: Interface Realisms: The Interface as Aesthetic Form (2005), http://pmc.iath.virginia.edu//issue.105/15.2pold.html

9. Seo, J.: Reading the Look and Feel: Interface Design and Critical Theories. Technical Report, MIT, Cambridge, Massachusetts (2001) 